\title{
Modification of initial growth and magnetism in $\mathrm{Fe} / \mathrm{Cu}(100)$
}

\author{
K. L. Man, ${ }^{1}$ W. L. Ling, ${ }^{2}$ Silena Y. Paik, ${ }^{2}$ H. Poppa,${ }^{3}$ M. S. Altman, ${ }^{1}$ and Z. Q. Qiu ${ }^{2}$ \\ ${ }^{1}$ Department of Physics, Hong Kong University of Science and Technology, Clear Water Bay, Kowloon, Hong Kong \\ ${ }^{2}$ Department of Physics, University of California at Berkeley, Berkeley, California 94720 \\ ${ }^{3}$ National Center for Electron Microscopy, Lawrence Berkeley National Laboratory, Berkeley, California 94720
}

(Received 16 July 2001; published 12 December 2001)

\begin{abstract}
The influence of the deposition rate upon the initial growth morphology and magnetism of ultrathin Fe films on the $\mathrm{Cu}(100)$ surface has been studied with low-energy electron diffraction, reflection high-energy electron diffraction, surface magneto-optical Kerr effect (SMOKE), and spin-polarized low-energy electron microscopy (SPLEEM). Dramatic changes in the first diffraction intensity oscillation during growth at room temperature indicate that simultaneous growth of the first and second atomic layers is converted to more perfect layer-bylayer growth when the deposition rate is reduced below $0.5 \mathrm{ML} / \mathrm{min}$. SPLEEM and SMOKE demonstrate the sensitivity of magnetism in $\mathrm{Fe} / \mathrm{Cu}(100)$ to details of interface formation during the initial growth in this range of deposition rates. Kinetic and thermodynamic mechanisms that relate to place exchange at the interface are discussed as the source of the observed growth and magnetic behavior.
\end{abstract}

DOI: 10.1103/PhysRevB.65.024409 PACS number(s): 75.70.Ak, 68.35.Fx, 68.37.Nq, 68.55.Jk

\section{INTRODUCTION}

Face-centered-cubic (fcc) Fe is one of the most interesting systems for studying the origin of magnetism due to the possibility of diverse magnetic phases it may manifest. Bulk Fe at room temperature has body-centered-cubic (bcc) structure and is ferromagnetic. fcc-phase bulk Fe exists only above $1184 \mathrm{~K}$. As this temperature is excessively high for magnetic ordering, it was impossible to study the magnetic properties of fcc Fe. Nonetheless, the close match between the lattice constants of fcc $\mathrm{Fe}$ and $\mathrm{Cu}$ has led to the idea of stabilizing $\mathrm{fcc} \mathrm{Fe}$ at low temperature as epitaxial thin films grown on $\mathrm{Cu}(100){ }^{1,2}$ The fcc $\mathrm{Fe} / \mathrm{Cu}(100)$ system has since then continuously attracted great interest in the investigation of magnetism.

While it was generally recognized that up to $\sim 11 \mathrm{ML}$ fcc Fe film can be stabilized on $\mathrm{Cu}(100)$, early experiments showed seemingly "contradictory" results on the magnetic phases of fcc Fe films., ${ }^{3,4}$ This contradiction was later resolved to have come from the different growth conditions: low-temperature growth of an fcc $\mathrm{Fe}$ film on $\mathrm{Cu}(100)$ results in the ferromagnetic (FM) phase alone, while the roomtemperature-grown Fe film exhibits the following rich magnetic phases. Below $5 \mathrm{ML}$, the Fe film is FM. Between 5 and $11 \mathrm{ML}$, the Fe film is antiferromagnetic (AFM) except that the top two layers at the vacuum interface are ferromagnetic. These two ferromagnetic layers are often refereed to as "magnetic live layers" and their magnetic moment in zero magnetic field is oriented perpendicular to the film surface $^{5-7}$ Besides, characteristic surface reconstructions found associated with these different magnetic phases ${ }^{8}$ suggest that magnetism in this system is strongly correlated with its structural properties. Detailed structural analysis using low-energy electron diffraction (LEED) shows that the FM phase is associated with the tetragonally distorted facecentered-cubic (fct) structure resulting from expansion of the fcc lattice in the normal direction of the film, while the AFM phase is associated with the fcc structure. ${ }^{9}$ Lattice distortion (both in- and out-of-plane) was also found to have a critical effect on the magnetic anisotropy of this system. ${ }^{10}$

These rich properties indicate that fcc $\mathrm{Fe}$ on $\mathrm{Cu}(100)$ may just be at a magnetic instability point. In fact, great effort has been made in theoretical calculations to explore the various possible magnetic phases of fcc Fe before the experimental realization of epitaxial fcc Fe films. Band structure calculations showed that fcc $\mathrm{Fe}$ has multiple magnetic phases in contrast with bcc Fe. ${ }^{11}$ For $r_{\mathrm{Ws}}$ (Wigner-Seitz radius) between 2.3 and 2.8 a.u., the total energy for bcc Fe has only one minimum, corresponding to a ferromagnetic state. On the other hand, the total energy for fcc Fe has two minima, corresponding to low-spin (LS) and high-spin (HS) states, respectively. The instability point between the LS and HS states occurs at $r_{\mathrm{WS}}=2.66$ a.u. As the Wigner-Seitz radius is 2.652 a.u. for fcc $\mathrm{Fe}$ and 2.667 a.u. for $\mathrm{Cu}$, it seems plausible to attribute the different magnetic phases to different structural phases. Total energy calculations have indeed confirmed the existence of both the FM and AFM phases in $\mathrm{Fe} / \mathrm{Cu}(100)$ under various conditions. ${ }^{12-14}$ However, due to the small energy difference between different phases and also the limitation of theoretical computations in taking into account realistic complications such as film roughness or interlayer mixing, it is difficult to predict exactly which magnetic phase will be favored over the others at critical points. Despite these reservations, current theory has advanced to deal with both $\mathrm{Fe}$ and $\mathrm{Cu}$ as a whole in the $\mathrm{Fe} / \mathrm{Cu}(100)$ system with first-principles total energy calculation. For example, different spin configurations have been proposed within the AFM phase in the 5-11 ML Fe thickness range. ${ }^{15}$ Parallel to the $a b$ initio calculation that usually deals with zero-temperature properties, spin dynamics based on phenomenological models are also considered to explain the unusual properties of the $\mathrm{Fe} / \mathrm{Cu}(100)$ system, especially the AFM phase. ${ }^{16}$

Although there have been many successful developments, the magnetic phases and their origins in $\mathrm{Fe} / \mathrm{Cu}(100)$ still remain a controversial and active field in thin-film magnetism research. A particularly important question is have we discovered all possible magnetic phases of fcc Fe? If not, what 
different conditions do we need to manifest those new phases? To answer these questions, experimental effort has been made to synthesize fcc $\mathrm{Fe}$ thin films under different conditions. This includes the growth of $\mathrm{FeNi}$ alloy on $\mathrm{Cu}(100),{ }^{17}$ the growth of fcc $\mathrm{Fe}$ on (100) substrates with lattice parameter larger or smaller than that of $\mathrm{Cu},{ }^{18-20}$ and the growth of fcc Fe on FM substrates such as $\mathrm{Ni}(100)$ and $\mathrm{Co}(100),{ }^{21,22}$ etc. A number of new features were discovered. For instance, magnetic live layers exist in $\mathrm{Fe}$ films in the thickness range 5-11 ML grown on $\mathrm{Co}(100)$ and $\mathrm{Ni}(100)$ substrates, but are located at both the surface and the interface. ${ }^{23,24}$ The magnitude of the magnetic moment of $\mathrm{Fe}$ is also different from that of $\mathrm{Fe}$ grown on $\mathrm{Cu}(100){ }^{25}$

The growth of $\mathrm{Fe} / \mathrm{Cu}(100)$ has also been a source of considerable controversy. First of all, it was noted that $\mathrm{Fe} /$ $\mathrm{Cu}(100)$ does not meet the necessary thermodynamic condition for layer-by-layer growth. ${ }^{26}$ That is, the change of surface energy, $\Delta \sigma=\sigma_{\mathrm{Fe}}+\sigma_{\mathrm{Cu}-\mathrm{Fe}}-\sigma_{\mathrm{Cu}}+\sigma_{e}$, should be negative if a continuous film is to be thermodynamically stable, where $\sigma_{\mathrm{Cu}}$ and $\sigma_{\mathrm{Fe}}$ are the surface energies of $\mathrm{Cu}$ and $\mathrm{Fe}$, respectively, $\sigma_{\mathrm{Cu}-\mathrm{Fe}}$ is the interface energy, and $\sigma_{e}$ is the elastic strain energy in the film, which is positive and an increasing function of thickness. In fact, the strain energy for fcc $\mathrm{Fe}$ on $\mathrm{Cu}(100)$ is expected to be small. ${ }^{27}$ The condition that best describes the $\mathrm{Fe} / \mathrm{Cu}(100)$ system, $\Delta \sigma \geqslant 0$, favors the formation of three-dimensional islands directly on the bare substrate (Volmer-Weber growth). Estimates of the surface and interface energies indicate that $\sigma_{\mathrm{Fe}}>\sigma_{\mathrm{Cu}-\mathrm{Fe}}+\sigma_{\mathrm{Cu}}{ }^{26,28}$ Given this energetic relationship, the system may prefer to adopt a $\mathrm{Cu} / \mathrm{Fe} / \mathrm{Cu}$ sandwich structure. The sandwich structure has indeed been observed for room-temperaturedeposited films, but only after application of a special annealing treatment following growth. ${ }^{28}$ Intermixing and segregation are particularly severe at all stages of growth above about $400 \mathrm{~K}^{29-33}$ The fact that continuous Fe films can be grown routinely at room temperature that are not fully terminated with $\mathrm{Cu}$ is a clear indication of kinetic limitations during growth. However, the kinetics of the initial growth during the formation of the $\mathrm{Fe} / \mathrm{Cu}$ interface is not well understood.

One aspect of the growth at room temperature that has been hotly debated is the initial growth morphology. It was argued on the basis of breakpoints in Auger electron spectroscopy (AES) that growth proceeds from the outset monoatomic layer by layer. ${ }^{1,34-37}$ However, the breakpoints were associated with the completion of atomic bilayers rather than monolayers by considering AES together with Rutherford backscattering (RBS) measurements. ${ }^{31}$ Complex initial growth at room temperature is also indicated by unusual diffraction intensity oscillations. In particular, the point at which the first continuous atomic monolayer is completed has been brought into question by the form of the first diffraction intensity oscillation, which is most often reported to be strongly suppressed or absent, ${ }^{5,27,33,38-41}$ although a full oscillation has also been observed. ${ }^{6}$ Although a robust diffraction oscillation peak corresponding to near-perfect completion of the second monolayer is always seen, the subsequent third oscillation is sometimes regular, sometimes not, or in between. It is widely agreed that regular oscilla- tions with $1 \mathrm{ML}$ periodicity return with the completion of the fourth layer. The diffraction intensity oscillation behavior has been alternatively interpreted in terms of bilayer growth or agglomeration of three-dimensional islands. These growth models are consistent with angular distributions in AES (Ref. 32) and x-ray photoelectron spectroscopy (XPS) ${ }^{29} \mathrm{CO}-$ titration experiments confirm the persistence of exposed $\mathrm{Cu}$ beyond $1 \mathrm{ML} \mathrm{Fe}$ coverage that is implicit in the bilayer and agglomeration models. ${ }^{29}$

The complexity of the initial growth was confirmed by real-space measurements with scanning tunneling microscopy (STM) ${ }^{42-47}$ low-energy ion scattering (LEIS), and impact collision ion-scattering spectroscopy (ICISS). ${ }^{48,49} \mathrm{Al}-$ though distinct monolayer height islands were seen initially with STM, the onset of growth in the second layer occurred well before completion of the first layer. Quantitative STM measurements of layer populations during simultaneous growth of the first and second layers have accounted for the attenuation of the first diffraction oscillation. ${ }^{44,45}$ Clear evidence of intermixing was also found with STM and LEIS where deposited $\mathrm{Fe}$ atoms become incorporated into the substrate initially by exchanging with $\mathrm{Cu}$ atoms in the topmost layer. Heterogeneous nucleation of $\mathrm{Cu}$ and $\mathrm{Fe}$ islands at the Fe inclusions was also observed with STM. These real-space measurements also confirm that growth of the third layer is initiated on a nearly complete two-layer film, despite the complexity of interface formation.

The initial growth morphology should be of significant interest because interface quality is known to influence magnetic properties, such as giant magnetoresistance, exchange coupling, and surface anisotropy. In particular, intermixing is a common problem that is confronted in magnetic thin-film growth. However, no systematic investigation of the effect of the initial growth on the magnetic properties of thermally evaporated $\mathrm{Fe} / \mathrm{Cu}(100)$ films has been carried out, since a similar trend of the Fe phases as a function of Fe film thickness has been observed in previous work. On the other hand, deposition of $\mathrm{Fe} / \mathrm{Cu}(100)$ by laser ablation, which gives a high instantaneous deposition rate, was found to have a significant impact upon the initial growth and magnetism. ${ }^{50}$ The aim of the present work is to systematically vary the initial growth of thermally evaporated $\mathrm{Fe} / \mathrm{Cu}(100)$ films and to determine whether this has any impact upon magnetism. We concentrate on the opposite extreme of laser ablation: that is, very low deposition rates extending below $0.5 \mathrm{ML} / \mathrm{min}$, which have seldom been used in prior work. Alternatively, variation of the deposition temperature within a small range of room temperature is expected to have a similar effect on the growth. Thus, the deposition rate dependent measurements described here also effectively describe small variations of the room-temperature deposition condition that must occur from laboratory to laboratory. LEED and reflection high-energy diffraction (RHEED) were used here to monitor growth, and Fe film magnetism was probed with surface magneto-optical Kerr effect (SMOKE) and spin-polarized low-energy electron microscopy (SPLEEM). We report that the initial growth morphology is strongly dependent upon the deposition rate and different initial growth is correlated with differences in magnetic properties, such as the SMOKE sig- 


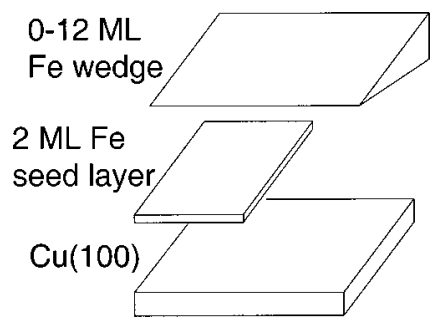

FIG. 1. Schematic of sample for SMOKE measurements. A seed layer of $2 \mathrm{ML} \mathrm{Fe}$ was grown at low rate $(\sim 0.06 \mathrm{ML} / \mathrm{min})$ on half of the $\mathrm{Cu}(100)$ crystal followed by a uniform $\mathrm{Fe}$ wedge of $0-12 \mathrm{ML}$ perpendicular to the [011] direction grown at high rate $(\sim 0.6 \mathrm{ML} /$ min). The $\mathrm{Cu}$ substrate was kept at room temperature during growth.

nal, coercivity, and Curie temperature of the live layer phase.

\section{EXPERIMENTAL DETAILS}

The experiments were performed on two different $\mathrm{Cu}(100)$ crystals using three experimental apparatuses. The LEED and SPLEEM experiments were carried out on the same crystal in two separate instruments, and the RHEED and SMOKE measurements were carried out together in the same instrument on the second crystal.

\section{A. RHEED and SMOKE}

The experiments were performed on a $\mathrm{Cu}(100)$ substrate in an ultrahigh-vacuum system at UC Berkeley equipped with quadrupole mass spectrometer, LEED, RHEED, AES, and SMOKE. The base pressure of the system was 7 $\times 10^{-11}$ Torr and remained below $2 \times 10^{-10}$ Torr during sample growth. The $\mathrm{Cu}(100)$ substrate was electropolished in air and cleaned in vacuum with cycles of Ar ion sputtering at $\sim 2 \mathrm{keV}$ and annealing at $\sim 600^{\circ} \mathrm{C}$ until a sharp $(1 \times 1)$ LEED pattern was obtained. Surface cleanliness was checked by AES.

Fe was evaporated from an alumina crucible wrapped with tungsten filament while the $\mathrm{Cu}$ substrate was kept at room temperature. The evaporation rate was monitored by a quartz oscillator located at the sample position and was also calibrated by RHEED oscillations. The typical low Fe evaporation rate was $0.017-0.06 \mathrm{ML} / \mathrm{min}$ and the high Fe evaporation rate was $0.33-0.83 \mathrm{ML} / \mathrm{min}$. Fe growth was monitored by RHEED oscillations for low and high growth rates.

To study the effect of the different initial growth on the properties of the subsequent films, we prepared the following sample on $\mathrm{Cu}(100)$ to avoid effects of substrate inhomogeneity and discrepancies in growth conditions. First we evaporated $2 \mathrm{ML}$ of $\mathrm{Fe}$ at a low rate $(\sim 0.06 \mathrm{ML} / \mathrm{min})$ as a seed layer on half of the substrate. Then an Fe wedge of $0-12 \mathrm{ML}$ was grown at a high rate $(\sim 0.6 \mathrm{ML} / \mathrm{min})$ on the entire $\mathrm{Cu}(100)$ substrate. In this way, we have two identical highrate-grown $\mathrm{Fe}$ wedges on each half of the $\mathrm{Cu}(100)$ substrate: one with a $2 \mathrm{ML}$ low-rate-grown Fe seed layer and the other not (Fig. 1). A study of these two wedges will thus single out the effect of the initial growth due to different evaporation rates.
The magnetic properties of the Fe films were studied by in situ SMOKE measurements in the temperature range of $100-300 \mathrm{~K}$. The sample was placed in the gap of two pairs of electromagnets which can apply magnetic field either perpendicular or parallel to the sample surface. A linearly polarized He-Ne laser was used as the light source. The intensity of the reflected beam from the sample surface was measured with a photodiode as a function of the applied magnetic field. A 1/4 waveplate and a polarizer were placed in front of the photodiode, with the polarizer $1^{\circ}$ away from the extinction. Hysteresis loops obtained by this SMOKE apparatus can be found in our earlier papers. ${ }^{22}$

\section{B. LEED and SPLEEM}

The LEED and SPLEEM experiments were performed on the same $\mathrm{Cu}$ crystal, but in two separate UHV systems located at the Hong Kong University of Science and Technology and the Lawrence Berkeley National Laboratory, respectively. The surface of the $\mathrm{Cu}$ sample was oriented parallel to the (100) plane to within $0.1^{\circ}$. It was cleaned by repeated cycles of Ar ion sputtering at $0.5 \mathrm{kV}$, and annealing to $875 \mathrm{~K}$. This procedure yielded a well-ordered surface with large terraces separated by monatomic height steps and that gave rise to sharp, instrumentally limited LEED spots with low background. ${ }^{51}$ Sample cleanliness was checked prior to each experiment with AES. Sample temperature was monitored by a $\mathrm{W}-3 \% \mathrm{Re} / \mathrm{W}-25 \% \mathrm{Re}$ thermocouple attached to the side of the sample. Furthermore, a type-N thermocouple was attached to the sample holder in close proximity to the sample in the SPLEEM. The type-N thermocouple has better lowtemperature thermovoltage characteristics than the W-Re thermocouple and was used as calibration during experiments below room temperature. Fe was deposited from a rod that was electron beam heated in the LEED and resistively heated in the SPLEEM experiments. The pressure rose to the mid- $10^{-10}$ Torr range during deposition from a base pressure of about $1-2 \times 10^{-10}$ Torr.

The LEED measurements were performed in a conventional low-energy electron microscope (LEEM). The LEEM was operated under conditions that give an illuminated area on the sample of $6 \mu \mathrm{m}$ and LEED transfer width ${ }^{52}$ of about $65 \mathrm{~nm}$. The contrast, resolution, and operation principle of the LEEM and SPLEEM have been described previously. ${ }^{51,53-56}$ The conventional LEEM diffraction and phase contrast mechanisms are augmented by magnetic contrast when a spin-polarized electron beam is used to illuminate the surface. Magnetic sensitivity of SPLEEM is based upon spin-dependent exchange scattering. The exchange asymmetry is defined as

$$
A_{\mathrm{ex}}=(1 / P)\left(I_{+}-I_{-}\right) /\left(I_{+}+I_{-}\right),
$$

where $I_{+}$and $I_{-}$represent the reflected intensities for oppositely polarized incident beams, called spin-up and spindown, and $P$ is the degree of incident beam polarization. Subtraction of spin-up and spin-down images in the numerator eliminates nonmagnetic diffraction and topographical image features that are found in conventional LEEM images and leaves features that originate exclusively in the magne- 
tism of the sample. Division by the spin-up and spin-down image sum yields an asymmetry image in which intensities are proportional to the incident beam spin polarization and the component of the local magnetization vector that lies along the incident beam polarization direction. The SPLEEM used in the present investigations is a compact flange-on instrument ${ }^{57}$ equipped with a spin manipulator ${ }^{58}$ and sample cooling. ${ }^{59}$ The spin manipulator can be used to adjust the azimuthal and polar angles of the incident beam spin direction fully relative to its propagation direction. This allows complete characterization of the sample magnetization direction in the in- and out-of-plane directions and tilted directions in between.

An important part of this work was the development of a new approach to monitor film thickness and magnetism simultaneously with SPLEEM. ${ }^{51}$ This approach exploits the fact that LEEM imaging of crystalline surfaces is fundamentally based upon diffraction. In particular, the sum of the spin-up and spin-down images that is found in the denominator of the exchange asymmetry [Eq. (1)] contains only diffraction information and no magnetic information. The well-known diffraction intensity oscillations that accompany periodic nucleation, growth, and completion of layers are obtained simply by integrating the intensity in the sum image. By monitoring diffraction intensity oscillations and the evolution of the exchange asymmetry simultaneously, continuously, and in the identical sample area during growth with SPLEEM, the film thickness and magnetism can be correlated with great precision.

\section{RESULTS}

\section{A. Growth}

The dependence of $\mathrm{Fe} / \mathrm{Cu}(100)$ growth upon deposition rate was studied by LEED spot profile and RHEED intensity measurements. LEED line scans were recorded continuously during growth in the [010] and [011] and equivalent directions for the (00) and (10)-equivalent diffraction spots. The measurements were performed at $24 \mathrm{eV}$ and normal incidence. The LEED spot peak intensity was determined by fitting the profiles with a Gaussian function without deconvolution of the instrumental function. The results of these measurements are shown in Fig. 2 for the range of deposition rate from 0.056 to $2.0 \mathrm{ML} / \mathrm{min}$. The deposition rates were determined from the oscillations corresponding to the fourth layer onward. The peak intensity plots in Fig. 2(b) are normalized to the same initial intensity.

The diffraction intensity oscillation that should correspond to the completion of the first atomic layer was strongly suppressed during growth at $0.5 \mathrm{ML} / \mathrm{min}$ and higher. Very strong oscillations are also observed near the completion of the second and third atomic layers, which are followed by a fourth and subsequent oscillations with diminished amplitude. Except for the form of the third oscillation, for which there is no general consensus, this reproduces the results that have been observed several times previously by various other diffraction techniques for rates in the same range. The resumption of regular oscillations at 4 ML has been attributed to the structural transition from the fct to the fcc
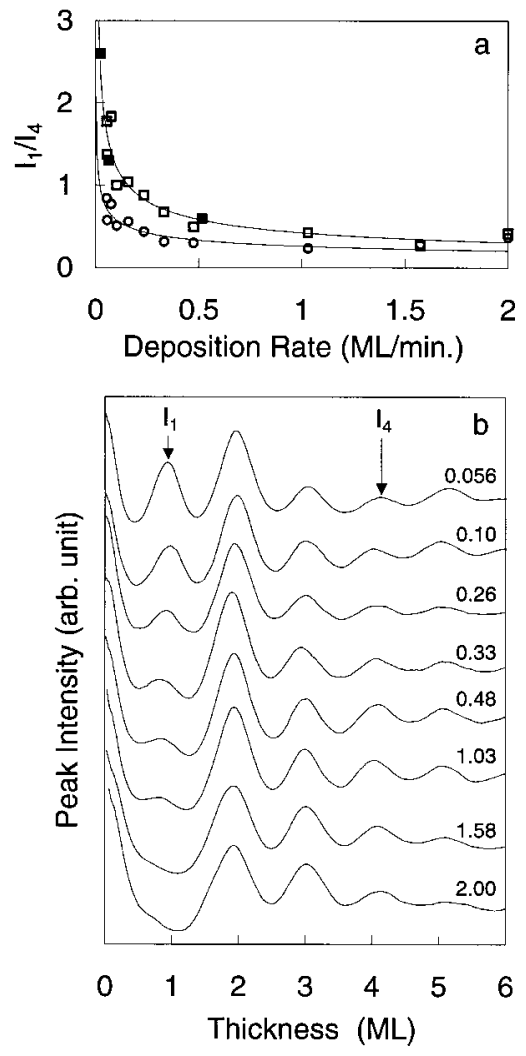

FIG. 2. (a) Amplitude of the first LEED intensity oscillation $I_{1}$, normalized to the fourth oscillation intensity $I_{4}$, vs deposition rate for the (00) $(\bigcirc)$ and (10) $(\square)$ spots. The RHEED results for $I_{1} / I_{4}$ are also indicated $(\mathbf{\square})$. The solid lines through the data points are power-law fits: $I_{1} / I_{4}=A$ rate $^{-n}$, where $A=0.42$ and 0.26 and $n=0.46$ and 0.35 for the (10) and (00) spots, respectively. (b) Oscillations of the (10) diffraction spot peak intensity at $24 \mathrm{eV}$ during growth of $\mathrm{Fe} / \mathrm{Cu}(100)$ at various deposition rates at $300 \mathrm{~K}$. The rates $(\mathrm{ML} / \mathrm{min})$ are indicated on the right-hand side.

phase. ${ }^{5,6}$ However, we also find that the first diffraction oscillation appears and is enhanced systematically as the rate is reduced below $0.5 \mathrm{ML} / \mathrm{min}$. It should be noted that the first oscillation peak occurs before the equivalent of $1 \mathrm{ML}$ of $\mathrm{Fe}$ has been deposited, but shifts gradually in the direction of the $1 \mathrm{ML}$ position as the deposition rate is reduced, with an apparent power law dependence upon rate. These trends are observed in both the (00) and (10) beams. The ratio of the peak intensities at the first oscillation position (or extrapolated position in the case of the highest deposition rate), $I_{1}$, and the fourth position, $I_{4}$, are shown in Fig. 2(a). The solid lines through the data points are power-law fits. However, the physical significance of the power-law dependences of the first oscillation peak is unclear.

RHEED intensity measurements during Fe film growth at room temperature are shown in Fig. 3 for evaporation rates of $0.52,0.065$, and $0.025 \mathrm{ML} / \mathrm{min}$, respectively. At $0.52 \mathrm{ML} /$ min, the RHEED intensity exhibits irregular oscillations for the first three monolayers and resumes regular oscillations at the fourth monolayer, related to the fct-to-fcc transition. As the evaporation rate is lowered, this transition does not change, but the RHEED intensities of the first three peaks, especially the first one, change dramatically. The ratio of the 


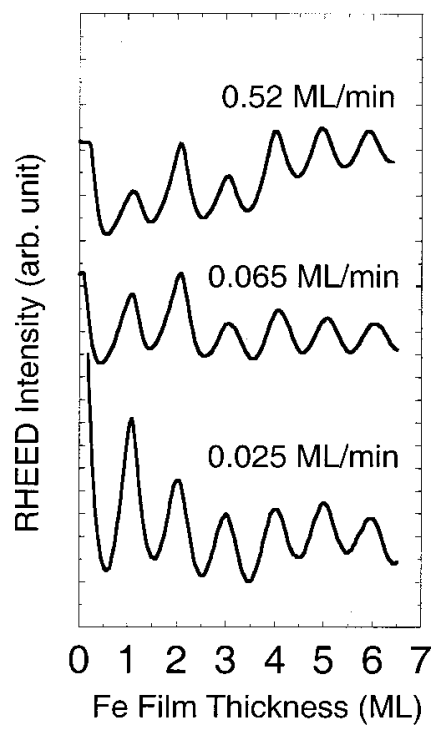

FIG. 3. RHEED intensity oscillations for Fe grown on $\mathrm{Cu}(100)$ at room temperature at various deposition rates. For comparison, the intensities are normalized to have equal intensity for the fourth oscillation.

intensity of the first peak to that of the fourth peak varies with the deposition rate, as indicated in Fig. 2(a), in very good agreement with changes observed with LEED. It should be mentioned that the peak intensities in RHEED oscillations depend on the incident angle of the electron beam. We kept the same incident angle (with the error less than one degree) for all RHEED oscillation measurements so that the first peak intensity variation in Fig. 3 is not due to the incident angle variation.

The dramatic changes of the LEED and RHEED intensity oscillations that occur when the rate is reduced below 0.5 $\mathrm{ML} / \mathrm{min}$ have not been reported before. In fact, deposition rates below $0.5 \mathrm{ML} / \mathrm{min}$ have seldom been used to grow $\mathrm{Fe} / \mathrm{Cu}(100)$, and there certainly has not been any systematic study of the rate dependence of the growth. Although some details of the diffraction oscillations are expected to differ between measurements made by different techniques, our independent LEED and RHEED results are consistent below $0.5 \mathrm{ML} / \mathrm{min}$ and also agree qualitatively with earlier work using RHEED, medium energy electron diffraction (MEED), and helium atom scattering (HAS) for rates above $0.5 \mathrm{ML} /$ min. Therefore, the change that is seen below $0.5 \mathrm{ML} / \mathrm{min}$ is not an artifact of using very low- or high-energy electrons. The following can be said in consideration of the possible action of $\mathrm{C}$ or $\mathrm{O}$ contaminants in our experiments. The base pressure all through our RHEED experiments was below 2 $\times 10^{-10}$ Torr. $\mathrm{H}_{2}, \mathrm{CO}_{2}, \mathrm{CO}$, and $\mathrm{CH}_{4}$ were the main components of the residual gas and each had a partial pressure $<10^{-11}$ Torr, which amounted to $<0.01$ Langmuir (L)/ML in all our growth conditions. While $\mathrm{CH}_{4}$ has a negligible adsorption coefficient in this system, ${ }^{60} \mathrm{CO}$ drastically affects the growth of $\mathrm{Fe}$ on $\mathrm{Cu}(100))^{5,27}$ As little as $0.08 \mathrm{~L} / \mathrm{ML}$ of $\mathrm{CO}$ extends the fcc region to more than $30 \mathrm{ML}$ as interstitial incorporation of $\mathrm{C}$, promoted by the presence of $\mathrm{O}$, expands the Fe lattice and stabilizes the fcc phase. However, $\mathrm{CO}$ does not have a significant effect on the first monolayer growth

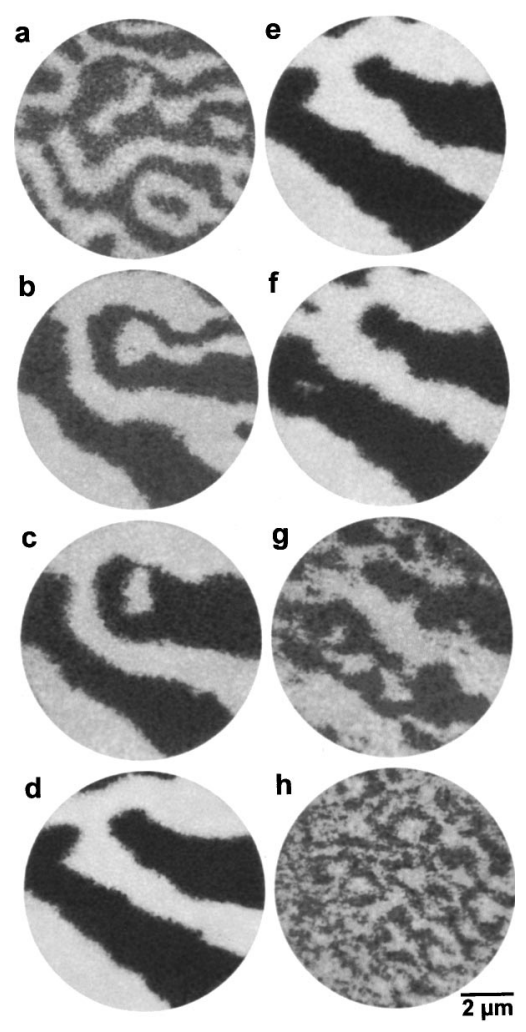

FIG. 4. SPLEEM magnetic difference images showing evolution of out-of-plane magnetic domains during growth of $\mathrm{Fe} / \mathrm{Cu}(100)$ at $300 \mathrm{~K}$ and deposition rate of $0.080 \mathrm{ML} / \mathrm{min}$ : (a) $2.13 \mathrm{ML}$, (b) 2.20 ML, (c) $2.33 \mathrm{ML}$, (d) $2.87 \mathrm{ML}$, (e) $3.13 \mathrm{ML}$, (f) $3.29 \mathrm{ML}$, (g) 3.60 $\mathrm{ML}$, and (h) $3.83 \mathrm{ML}$. Image contrast variations between images have not been altered. The imaging energy is $1.8 \mathrm{eV}$.

and $\mathrm{C}$ or $\mathrm{O}$ induces a $c(2 \times 2)$ surface reconstruction which was not observed after the growth in our experiments. We have also found that the initial growth was not altered by coadsorbed oxygen at pressures up to $1.0 \times 10^{-9}$ Torr in LEED experiments, although a strong oxygen-induced $c$ (2 $\times 2$ ) diffraction pattern was observed under these conditions. Similar tests on the influence of $\mathrm{H}$ adsorption were not made. Although $\mathrm{H}$ adsorption during growth does influence magnetic properties, ${ }^{61}$ no impact upon the initial diffraction intensity oscillations was reported. Therefore, the dramatic change of the first RHEED and LEED intensity oscillations is unlikely to be caused by $\mathrm{C}, \mathrm{O}$, or $\mathrm{H}$ contamination, but rather we attribute it directly to an intrinsic dependence of the initial growth processes upon the deposition rate.

\section{B. Magnetism}

We then proceed to investigate the effect of deposition rate on the magnetic properties of $\mathrm{Fe}$ films by performing SPLEEM measurements on films grown entirely at a fixed deposition rate. Magnetic imaging was performed with SPLEEM continuously during growth at various rates below $0.5 \mathrm{ML} / \mathrm{min}$, where significant changes in the initial growth were observed with LEED and RHEED. An example of the evolution of magnetic domain structure during growth is 

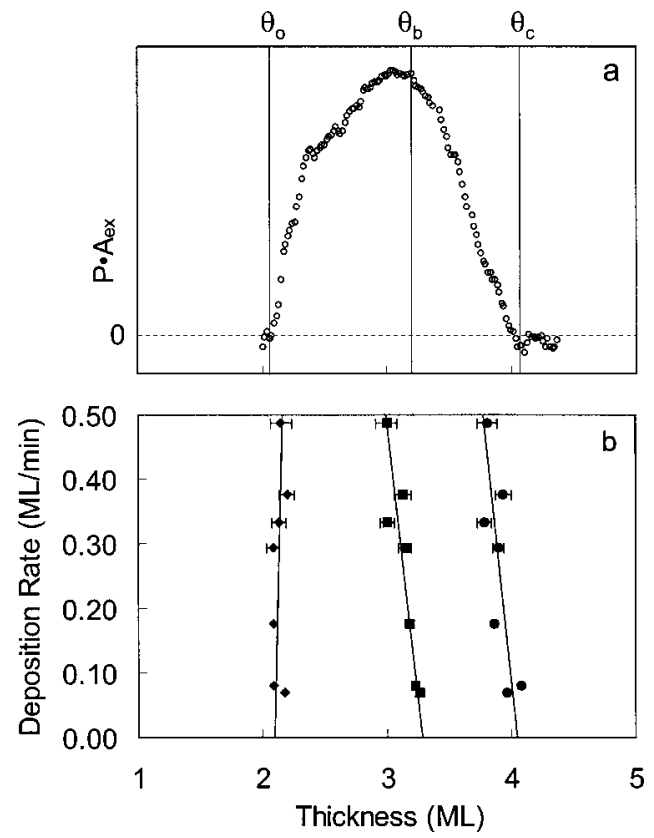

FIG. 5. (a) Exchange asymmetry [Eq. (1)] vs film thickness determined from in situ SPLEEM magnetic images during $\mathrm{Fe} /$ $\mathrm{Cu}(100)$ growth at $0.080 \mathrm{ML} / \mathrm{min}$ and $300 \mathrm{~K}$ (see Fig. 4). The vertical lines indicate the onset of magnetism $\left(\theta_{o}\right)$, break up of magnetic domains $\left(\theta_{b}\right)$, and complete disappearance of magnetism $\left(\theta_{c}\right)$ determined by inspection of images. (b) Dependence of $\theta_{o}$ $(\bullet), \theta_{b}(\boldsymbol{\square})$, and $\theta_{c}(\boldsymbol{\bullet})$ upon the deposition rate determined from SPLEEM images during growth of $\mathrm{Fe} / \mathrm{Cu}(100)$ at $300 \mathrm{~K}$.

shown in Fig. 4. This figure depicts the out-of-plane component of magnetization. No in-plane component was detected. Key observations were the absence of magnetism until its onset in the form of magnetic stripe domains slightly after 2 ML had been deposited, coarsening of magnetic domains until a steady configuration was reached, and breakup of magnetic domains into a continuously smaller domain configuration until the complete disappearance of magnetism. The onset $\theta_{o}$, breakup $\theta_{b}$, and completion $\theta_{c}$ coverages were noted accurately and are discussed further below. The variation of exchange asymmetry [see Eq. (1)] determined from SPLEEM magnetic contrast is shown in Fig. 5. The exchange asymmetry was found to increase sharply initially and then more slowly until reaching a plateau slightly above $3 \mathrm{ML}$, followed by a sharp decrease to zero. The point that the exchange asymmetry begins to decrease correlates with the breakup of magnetic domains, at $\theta_{b}$, which is observed visually. For example, a very small "bright" domain appears in the left-hand side of Fig. 4(f) at a coverage slightly larger than $\theta_{b}$, which is absent in the images Figs. 4(d) and 4(e) at lower coverage.

Under certain conditions, the exchange asymmetry [Eq. (1)] may be taken to be linearly proportional to the magnetization. ${ }^{51}$ Assuming this to be the case for $\mathrm{Fe} /$ $\mathrm{Cu}(100)$, these observations reproduce the generally accepted behavior of this system, which is the onset of ferromagnetism with out-of-plane easy axis at room temperature at a film thickness of about $2 \mathrm{ML}$ and transition to a live layer phase with Curie temperature below room temperature

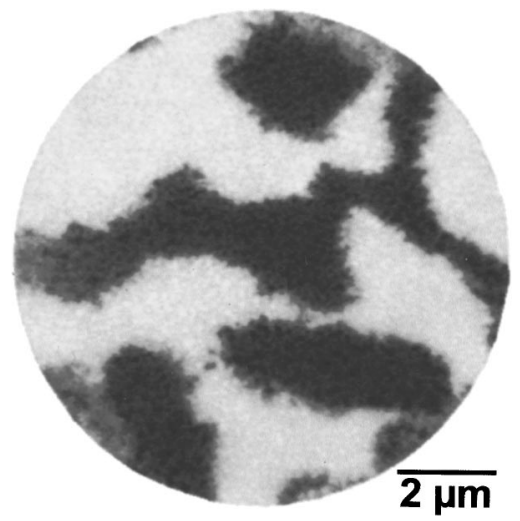

FIG. 6. SPLEEM image of the out-of-plane magnetic domain configuration for the live layer at $5.5 \mathrm{ML}$ film thickness at $T$ $=240 \mathrm{~K}$. The film was prepared at $300 \mathrm{~K}$ at a rate of $0.20 \mathrm{ML} / \mathrm{min}$. The imaging energy is $1.8 \mathrm{eV}$.

at higher thickness. Below $2 \mathrm{ML}$, the Curie temperature of the film is also below room temperature. ${ }^{62}$ The variation of the exchange asymmetry with increasing thickness that is determined with SPLEEM (Ref. 51) also resembles the change of the magnetic remanence that is observed with the SMOKE (see Refs. 5, 6, 9, and 61 and results presented below). However, the transitions distinguished by the onset, breakup, and completion coverages were found to depend slightly, yet systematically, upon the deposition rate (Fig. 5). Specifically, the reduction of the deposition rate causes a delay of the transition to the live layer phase, defined by $\theta_{b}$ and $\theta_{c}$ in Fig. 5, while the onset of ferromagnetism at room temperature is largely unaffected. The error bars in this figure represent twice the incremental coverage deposited between acquisitions of successive images.

The Curie temperature of the live layer was also determined with SPLEEM. Immediately after deposition of 5.5 $\mathrm{ML} \mathrm{Fe}$, as determined by diffraction intensity oscillations during growth, the sample was cooled well below the Curie temperature. This gave rise to well-defined out-of-plane magnetic domains (Fig. 6). Then the sample was warmed up slowly until the magnetic contrast disappeared. The sample temperature was then cycled slowly and within an increasingly smaller range until magnetic domains appeared and disappeared nearly reversibly at a temperature that was taken to be the Curie temperature. As shown in Fig. 7, the Curie temperature is generally suppressed for films grown at lower rates.

The SPLEEM measurements described above probe the influence of deposition rate of magnetism at all stages of growth. In order to focus more explicitly on the relationship between the initial growth and magnetism, thicknessdependent SMOKE measurements were performed at $100 \mathrm{~K}$ along the two adjacent Fe wedges with and without a $2 \mathrm{ML}$ $\mathrm{Fe}$ seed layer grown at low rate (see Fig. 1). For both wedges, the magnetization was perpendicular to the sample surface so that only polar hysteresis loops are reported here (see Fig. 8). It should be noted that although the wedge slope is well defined, there exists a systematic thickness error due to the registration of the wedge. From the SMOKE laser beam size $(\sim 0.3 \mathrm{~mm})$ and the slope of the wedge $(\sim 2-3$ $\mathrm{ML} / \mathrm{mm}$ ), we estimate the systematic thickness error is be- 


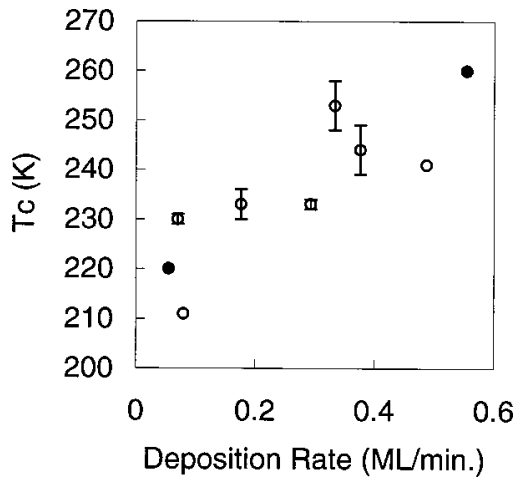

FIG. 7. Dependence of live layer Curie temperature upon the deposition rate determined by SPLEEM $(\bigcirc)$ and SMOKE $(\bullet)$. For the SMOKE data, the rate refers to films with low-growth-rate seed layer $(0.055 \mathrm{ML} / \mathrm{min})$ or without $(0.55 \mathrm{ML} / \mathrm{min})$. The total film thickness for SPLEEM data is $5.5 \mathrm{ML}$ and for SMOKE data is 7 ML.

tween -1 and 1 ML: i.e., the curves in Figs. 8 and 9 could shift in thinner or thicker directions by up to $1 \mathrm{ML}$ due to this systematic error. Since the SPLEEM measurement was done on a nonwedge sample whose thickness is determined by LEEM intensity oscillations, any thickness discrepancy between SMOKE and SPLEEM results should be attributed to the wedge registration error in the SMOKE sample.

Magnetic remanence and coercivity of the Fe films were measured along the two Fe wedges. The results are shown in Figs. 8 and 9 as a function of Fe film thickness. The general thickness dependences of the magnetic properties of the two samples are very similar and agree with the literature for molecular-beam-epitaxy- (MBE-) grown Fe films deposited at room temperature. ${ }^{6}$ The magnetic remanence increases with thickness for Fe films thinner than $4 \mathrm{ML}$, indicating the formation of the fct FM phase. The remanence drops to a much lower value at $\sim 4 \mathrm{ML}$ of Fe and remains at the low value with weak oscillations in the 5-11 ML range. This is the fcc phase of AFM Fe plus a 2 ML FM surface layer. ${ }^{5}$ The fct-fcc transition is accompanied by a cusp in the coercive field.

Despite the overall similarity of the magnetic phases of the two wedges, there are noticeable differences due to the initial deposition rate. First, the SMOKE remanence with the $2 \mathrm{ML} F e$ seed layer grown at a low rate is $\sim 30 \%$ lower than without the seed layer. Although the Kerr signal is propor-

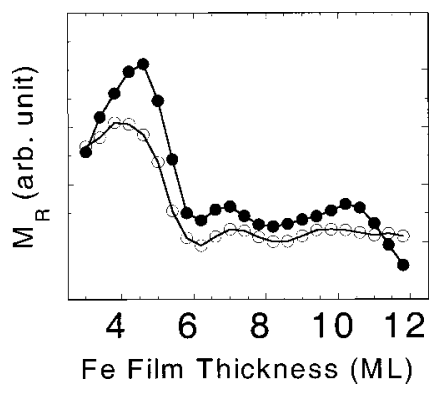

FIG. 8. SMOKE remanence $\left(M_{R}\right)$ along the Fe wedges with $(\bigcirc)$ and without $(\bigcirc)$ the 2 ML low-rate-grown seed layer.

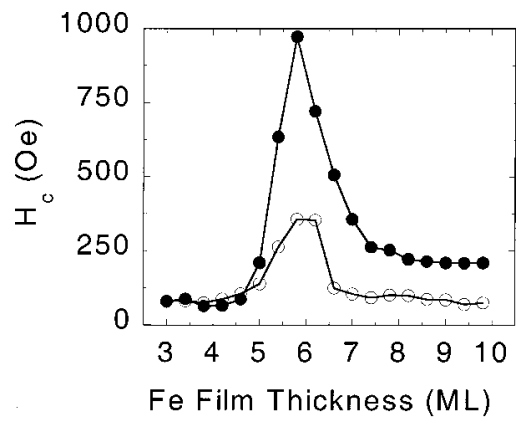

FIG. 9. The coercive field $H_{C}$ measured using SMOKE along the $\mathrm{Fe}$ wedges with a $2 \mathrm{ML}$ low-rate-grown seed layer present $(\mathrm{O})$ and with no 2 ML low-rate-grown seed layer present $(\mathbf{O})$.

tional to the magnetization, the proportionality coefficient depends on the optical properties of the film. On the other hand, in situ RHEED and LEED results indicate the great similarity of the Fe films with and without the seed layer. Therefore, it is unlikely that the $30 \%$ SMOKE signal difference is caused entirely by differences of the optical properties that might be effected by the initial deposition rate. The $30 \%$ reduction of the SMOKE signal suggests, instead, that the magnetization of the Fe film is reduced by the low initial growth rate. Second, the coercivity of the SMOKE loop with the seed layer is much smaller than that without the seed layer.

To ensure that the observed difference is inherent to the samples and not due to inhomogeneity of the substrate, we did SMOKE measurements near the border between the two Fe wedges. Because there is a $2 \mathrm{ML}$ thickness change across the border, we choose the live layer region (10 and $8 \mathrm{ML}$ for the wedges with and without the 2 ML seed layer, respectively) where there is no significant change of remanence and coercivity along each wedge. Figure 10 shows SMOKE loops at different positions across the border. We see clearly the transition from one wedge to the other. Hysteresis loops with consistent saturation fields and coercivity change suddenly as the SMOKE laser beam crosses the border. In par-

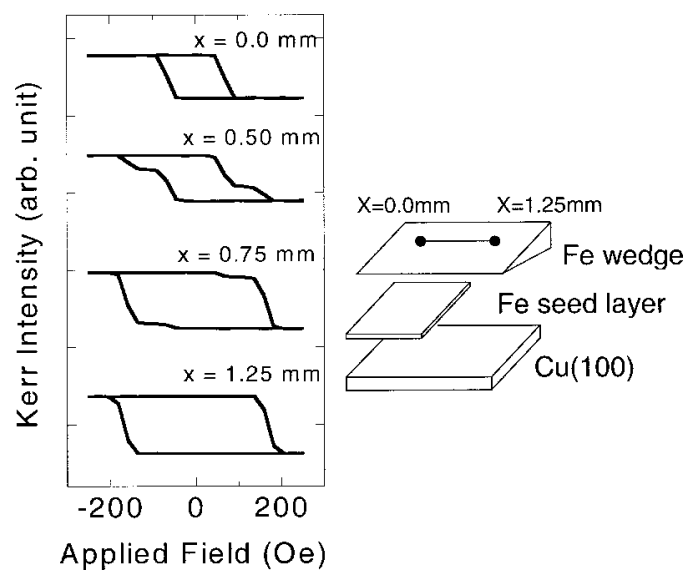

FIG. 10. Polar hysteresis loops at constant Fe wedge thickness of $8 \mathrm{ML}$ along the substrate crossing the boundary of the two samples with (top) and without (bottom) the low-rate-grown seed layer. 


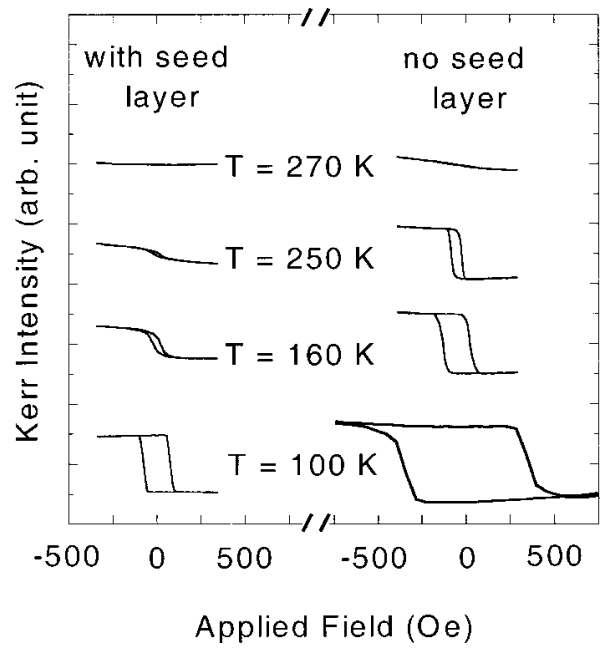

FIG. 11. Easy axis hysteresis loops at various temperatures of a 7 ML Fe film grown with (left) and without (right) the low-rategrown seed layer. The magnetic remanence disappears on the sample with seed layer at a lower temperature than on the one without, indicating a lower Curie temperature due to the seed layer.

ticular, the hysteresis loop becomes a superposition of two loops with different coercivities at the border due to the finite laser beam size $(\sim 0.3 \mathrm{~mm}$ at half maximum intensity). This result gives unequivocal proof that the difference in the magnetic coercivity in the two Fe wedges is not an artifact from substrate inhomogeneity, but is intrinsic to the initial growth rate.

The last difference we observed was the Curie temperature of the surface live layer. Motivated by the observation of the reduced magnetic remanence from low-rate initial growth, we performed temperature dependence measurements of the hysteresis loops for $7 \mathrm{ML} F$ (total thickness) with and without the seed layer. If the seed layer reduces the magnetization of the Fe film, then a lower Curie temperature would be expected. SMOKE loops at various temperatures are shown in Fig. 11. Once again, we find a remarkable difference in the Curie temperature of the two films. While the film of $7 \mathrm{ML} \mathrm{Fe}$ grown at high rate has a Curie temperature of $\sim 260 \mathrm{~K}$, the film with $5 \mathrm{ML} \mathrm{Fe}$ grown at high rate on top of 2 ML seed layer with low rate (total thickness $7 \mathrm{ML}$ ) has a lower Curie temperature of $\sim 220 \mathrm{~K}$. The SMOKE results are plotted in Fig. 7 with respect to the rates that the initial two layers were grown. These results are in close agreement with the correlation of the Curie temperature versus deposition rate observed with SPLEEM.

\section{DISCUSSION}

The initial growth morphology of $\mathrm{Fe} / \mathrm{Cu}(100)$ at room temperature has been clearly shown by diffraction intensity oscillations to depend upon the deposition rate. This phenomenon is qualitatively similar to the dependence upon deposition temperature that was noted previously. ${ }^{39}$ The strong suppression of the first diffraction oscillation that was observed here and previously ${ }^{5,27,33,38-41}$ during growth at room temperature with high deposition rates has already been accounted for by quantitative measurements of layer populations during simultaneous growth of the first and second layers with STM. ${ }^{44,45}$ We also find that the diffraction spots are broadened near the completion of nominally $1 \mathrm{ML}$ at high rates. This is consistent with the high surface defect density detected with LEIS (Ref. 48) and HAS and the short step separation seen with HAS (Ref. 41) at this stage of growth. The emergence and systematic enhancement of the first diffraction oscillation that occurs when the deposition rate is lowered signals that the first atomic monolayer grows more completely before significant nucleation and growth in the second layer.

The change of the initial growth morphology should be of significant interest because of the known relationship between interface quality and magnetic properties. However, the reasons for this change, and therefore the microscopic differences, if any, between interfaces formed at low and high deposition rates are unclear. Of course, suppression of premature growth in the second layer at lower deposition rate must mean that step-down motion from the second to the first growing layer is enhanced relative to nucleation in the second layer. This is understandable in a simple view because the nucleation rate should scale with the adatom concentration, which is generally diminished at lower deposition rate. Besides the arrival rate, the presence of a step edge diffusion barrier that inhibits step-down motion can influence the adatom concentration in the second layer, as well as morphological factors such as island shape and size. That is, step-down motion from small or irregular-shaped islands will be enhanced because of the greater number of times that an atom encounters a step, i.e., higher attempt frequency. A step edge diffusion barrier between the second and first layers has been invoked to explain changes of the initial growth morphology for $\mathrm{Co} / \mathrm{Cu}(100)$ that occur upon reduction of the deposition rate that are similar to those reported here for $\mathrm{Fe} / \mathrm{Cu}(100){ }^{63}$ Near-perfect completion of the second layer prior to growth in the third layer, seen for $\mathrm{Co} / \mathrm{Cu}(100)$ as well as for $\mathrm{Fe} / \mathrm{Cu}(100)$ at all deposition rates, was ascribed to the absence of a barrier to motion at homogeneous steps between the third and second layers. A step edge barrier may very well be present at the heterogeneous step between the second and first layers. However, this alone cannot explain the change of initial growth morphology that is observed upon reduction of the deposition rate. The reason is that growth at a lower rate occurs closer to equilibrium than at high rates. This means that the growth morphology must embody thermodynamics more so at lower deposition rates, not less. As mentioned above, three-dimensional island growth or possibly an inverted $\mathrm{Cu} / \mathrm{Fe}$ layer, not a complete Fe wetting monolayer, can be expected in consideration of surface and interface energetics.

An additional effect that could contribute to the change of the initial growth morphology may be that the number of attempts per unit time for step-down motion from the second to the first layer increases when the deposition rate is reduced. This could happen if the number of times that an atom diffusing in the second layer encounters a step is increased due to a higher island density or more irregular-island shapes, i.e., higher step density. However, the island nucleation rate conventionally decreases when the deposition rate 
is reduced or, equivalently, when the temperature is increased. Unconventionally, heterogeneous nucleation at sites where $\mathrm{Fe}$ has exchanged places with $\mathrm{Cu}$ in the topmost substrate layer provides a mechanism for the first-layer island density to increase anomalously at lower deposition rate. This is analogous to the increase of the island density that was found with increasing temperature for growth of $\mathrm{Ni}$ on $\operatorname{Ag}(111)$; simulations and rate equation analyses were used to demonstrate that place-exchange-mediated nucleation can result in an "inverted" dependence of the nucleation rate on temperature. ${ }^{64}$ According to Ref. 64, the usual island density scaling held at low temperature, while the onset of place exchange at higher temperature caused an anomalous increase of the island density. At intermediate temperatures, a distinct minimum of the island density was found at the crossover between conventional and place-exchangemediated nucleation regimes. Presumably, place exchange can cause the same anomalous increase of the island density when the deposition rate is reduced at fixed temperature, which is the situation that describes our experiments on $\mathrm{Fe} /$ $\mathrm{Cu}(100)$. Furthermore, place-exchange-mediated nucleation behavior may be even more pronounced for growth on fcc(100) surfaces where an exchange diffusion channel is available that does not exist on the (111) surface. There is already ample evidence of intermixing during the initial growth of $\mathrm{Fe} / \mathrm{Cu}(100)$ and heterogeneous nucleation at $\mathrm{Fe}$ inclusion sites. ${ }^{29,42,43,48,49}$ Place exchange and heterogeneous nucleation in the $\mathrm{Co} / \mathrm{Cu}(100)$ system have also been observed. ${ }^{63,65}$

The prevalence for place exchange and heterogeneous nucleation during the initial growth of $\mathrm{Fe} / \mathrm{Cu}(100)$ also suggests another mechanism for the observed growth behavior. To start with, $\mathrm{Cu}$ atoms that are released by place exchange with $\mathrm{Fe}$ at the outset of growth form islands in the first growing layer, and the edges of these $\mathrm{Cu}$ islands are then decorated with $\mathrm{Fe}$ during subsequent deposition. ${ }^{42,43}$ This is seen also for $\mathrm{Co} / \mathrm{Cu}(100)$ growth. ${ }^{63}$ Second-layer nucleation prior to completion of the first layer has also been clearly seen to be heterogeneous to some extent for $\mathrm{Fe} / \mathrm{Cu}(100){ }^{42,43}$ Therefore, suppression of second-layer nucleation at a low deposition rate may be a signal that the $\mathrm{Fe}$ content of the first layer is diminished. In other words, at low deposition rate, Fe exchanges more completely with $\mathrm{Cu}$ during the growth of the first layer. The driving force for this is the favorable energetics of Fe embedded in the topmost substrate layer rather than resting on top of it and of the inverted $\mathrm{Fe} / \mathrm{Cu}$ layer configuration. First-principles calculations have shown that an inverted $\mathrm{Fe} / \mathrm{Cu}$ layer is energetically favored over a completely wetting Fe monolayer. ${ }^{66}$ Taken to the opposite extreme of a very high deposition rate, one could expect that the place exchange process would be suppressed because Fe island nucleation occurs on a much faster time scale. Such experiments have been carried out already using laser ablation. ${ }^{50}$ Interestingly, the first diffraction intensity oscillation and layer-by-layer growth were seen very clearly in these experiments. The first oscillation was even more pronounced than observed here at the lowest deposition rates. Furthermore, the magnetic properties of films grown by laser ablation were quite distinct from those of films grown by thermal evapora- tion at rates discussed in this paper. It would therefore be worthwhile to check if the $\mathrm{Cu}$ content of the first layer depends upon the deposition rate in a manner suggested by the growth behavior presented here.

Magnetic transitions that occur during growth of $\mathrm{Fe} /$ $\mathrm{Cu}(100)$ at room temperature were found to depend subtly upon the deposition rate in the range $0.07-0.49 \mathrm{ML} / \mathrm{min}$, where significant changes of the initial growth morphology occur. Possible reasons for this subtle change, including interface mixing, surface roughness, and contamination arising from $\mathrm{Cu}$ segregation or $\mathrm{H}$ adsorption, have been considered. ${ }^{51}$ In particular, $\mathrm{H}$ adsorption is known to delay the fct-fcc structural transformation that drives the formation of the live layer. ${ }^{61}$ For this reason, adsorption of residual $\mathrm{H}$, which is expected to be more pronounced at a lower deposition rate, is considered to be a possible reason for the trend of $\theta_{b}$ and $\theta_{c}$ shown in Fig. 5. A recent STM work ${ }^{67}$ in the 2-4 ML thickness range with $1 \mathrm{ML} / \mathrm{min}$ deposition rate reveals that the ferromagnetic phase is associated with zigzag bcc-like stripes and that the conversion of the bcc-like stripe to fcc structure occurs at the fct-to-fcc transition. This result implies that the strain relaxation at the fct-to-fcc transition needs to be aided by magnetic energy. It also suggests a likely reason why both the magnetization and fct-to-fcc transition are modified by the deposition rate in our work. Therefore, it would be really interesting to see the STM results in the future for a low deposition rate.

The impact of the initial growth upon magnetism was found to be more significant in SMOKE measurements of magnetic remanence, coercivity, and live layer Curie temperature. The Curie temperature is first of all associated with the strength of the coupling constants between spins. On the other hand, the disparity in the Curie temperature and magnetic remanence for wedges with and without a 2 ML seed layer grown at a low rate suggests that these phenomena originate in a difference of the intrinsic value of the magnetization of the two films. SPLEEM measurements also reveal that the live layer Curie temperature is suppressed when growth is carried out at lower deposition rate. It is tempting to attribute this trend in SPLEEM investigations also to $\mathrm{H}$ adsorption, although there has been no prior report of such an effect. However, despite the different approaches to film preparation employed, the agreement between SPLEEM and SMOKE results shown in Fig. 7 suggests that the deposition rate during the initial growth is a key factor that determines the live layer Curie temperature.

Finally, due to the clear impact of the initial growth upon the live layer magnetism, we wish to remark on the oscillations in the magnetic remanence in the 5-11 ML thickness range that are seen in Fig. 8. These oscillations originate from an AFM phase of the Fe film below the surface live layer. ${ }^{6}$ It is interesting to note that the oscillation periodicity is $\sim 2.6 \mathrm{ML}$ of Fe instead of $2 \mathrm{ML}$, showing that AFM Fe has a nontrivial spin configuration as in ordinary antiferromagnetism. This result is consistent with Ref. 6. Although many spin configurations have been proposed theoretically in the AFM phase of fcc Fe, ${ }^{15}$ the individual configuration always gives an integral number of monolayers in the oscillation periodicity in magnetization. As there is very little energy 
difference between the different spin configurations, we speculate that the observed 2.6 ML oscillation periodicity may be a result of the coexistence of these many spin configurations or a result of nontrivial spin density waves. Nonintegral oscillation periodicity exists in other phenomena such as the oscillatory magnetic interlayer coupling where the formation of quantum well states generates the oscillatory coupling. ${ }^{68}$ However, quantum well states usually do not significantly modify the magnitude of magnetization. Therefore, it is unlikely that the oscillation in the magnetic remanence is due to the quantum well states. A final answer to this 2.6 ML oscillation periodicity requires further detailed study both experimentally and theoretically.

\section{CONCLUSION}

In conclusion, the initial growth of $\mathrm{Fe}$ on $\mathrm{Cu}(100)$ by thermal evaporation depends strongly on the deposition rate. Simultaneous growth of the first and second atomic layers is converted to more perfect layer-by-layer growth when the deposition rate is reduced below $0.5 \mathrm{ML} / \mathrm{min}$. A simple kinetic model in which step-down motion is enhanced at lower deposition rates, i.e., closer to equilibrium, is ruled out because a complete wetting Fe monolayer is thermodynamically unstable relative to three-dimensional clustering or an inverted $\mathrm{Cu} / \mathrm{Fe}$ configuration. Instead, kinetic and thermodynamic mechanisms that relate to $\mathrm{Fe}-\mathrm{Cu}$ place exchange at the interface are discussed as the source of the observed growth behavior. Further experiments are needed to verify the proposed mechanisms. The choice of deposition rate during the initial growth is also found to have a substantial impact upon the magnetic properties of $\mathrm{Fe}$ films. Interface formation at lower deposition rates induces the reduction of magnetic remanence, coercivity, and live layer Curie temperature. These results demonstrate the sensitivity of magnetism in $\mathrm{Fe} /$ $\mathrm{Cu}(100)$ to details of interface formation during the initial growth. This effect must be taken into account in a discussion of the magnetic properties of $\mathrm{Fe} / \mathrm{Cu}(100)$, especially with regard to the magnetism of the live layer phase and antiferromagnetism of the underlying layers which remain unresolved.

\section{ACKNOWLEDGMENTS}

We are grateful to J. Venables for helpful discussions. This work was supported in part by the DOE BES-MS under Contract No. DEAC03-76SF00098 at LBNL, NSF Grant No. DMR-0110034, and by the Hong Kong Research Grant Council under Grants No. HKUST690/96P and HKUST6128/97P.
${ }^{1}$ D. Pescia, M. Stampanoni, G. L. Bona, A. Vaterlaus, R. F. Willis, and F. Meier, Phys. Rev. Lett. 58, 2126 (1987).

${ }^{2}$ P. A. Montano, G. W. Fernando, B. R. Cooper, E. R. Moog, H. M. Naik, S. D. Bader, Y. C. Lee, Y. N. Darici, H. Min, and J. Marcano, Phys. Rev. Lett. 59, 1041 (1987).

${ }^{3}$ C. Liu, E. R. Moog, and S. D. Bader, Phys. Rev. Lett. 60, 2422 (1988)

${ }^{4}$ W. A. A. Macedo and W. Keune, Phys. Rev. Lett. 61, 475 (1988).

${ }^{5}$ J. Thomassen, F. May, B. Feldmann, M. Wuttig, and H. Ibach, Phys. Rev. Lett. 69, 3831 (1992).

${ }^{6}$ D. Li, M. Freitag, J. Pearson, Z. Q. Qiu, and S. D. Bader, Phys. Rev. Lett. 72, 3112 (1988).

${ }^{7}$ R. D. Ellerbroke, A. Fuest, A. Schatz, W. Keune, and R. A. Brand, Phys. Rev. Lett. 74, 3053 (1995).

${ }^{8}$ Pascal Xhonneux and Eric Courtens, Phys. Rev. B 46, 556 (1992)

${ }^{9}$ S. Müller, P. Bayer, C. Reischl, K. Heinz, B. Feldmann, H. Zillgen, and M. Wuttig, Phys. Rev. Lett. 74, 765 (1995).

${ }^{10}$ D. E. Fowler and J. V. Barth, Phys. Rev. B 53, 5563 (1996).

${ }^{11}$ V. L. Moruzzi, P. M. Marcus, K. Schwarz, and P. Mohn, Phys. Rev. B 34, 1784 (1986).

${ }^{12}$ F. J. Pinski, J. Staunton, B. L. Gyorffy, D. D. Johnson, and G. M. Stocks, Phys. Rev. Lett. 56, 2096 (1986).

${ }^{13}$ T. Kraft, P. M. Marcus, and M. Scheffler, Phys. Rev. B 39, 6957 (1989).

${ }^{14}$ S. S. Peng and H. J. F. Jansen, J. Appl. Phys. 69, 6132 (1991).

${ }^{15}$ T. Asada and S. Blügel, Phys. Rev. Lett. 79, 507 (1997).

${ }^{16}$ R. E. Camley and D. Li, Phys. Rev. Lett. 84, 4709 (2000).

${ }^{17}$ F. O. Schumann, R. F. Willis, K. G. Goodmann, and J. G. Tobin, Phys. Rev. Lett. 79, 5166 (2000).
${ }^{18}$ R. Rochow, C. Carbone, Th. Dodt, F. P. Johnen, and E. Kisker, Phys. Rev. B 41, 3426 (1990).

${ }^{19}$ D. J. Keavney, D. F. Storm, J. W. Freeland, I. L. Grigorov, and J. C. Walker, Phys. Rev. Lett. 74, 4531 (1995).

${ }^{20}$ D. Li, D. J. Keavney, J. Pearson, S. D. Bader, J. Page, and W. Keune, Phys. Rev. B 57, 10044 (1998).

${ }^{21}$ W. L. O'Brian and B. P. Tonner, Phys. Rev. B 52, 15332 (1995).

${ }^{22}$ E. Escorcia-Aparicio, R. K. Kawakami, and Z. Q. Qiu, Phys. Rev. B 54, 4155 (1996).

${ }^{23}$ X. Gao, M. Salvietti, W. Kuch, C. M. Schneider, and J. Kirschner, Phys. Rev. B 58, 15426 (1998).

${ }^{24}$ B. Schirmer and M. Wuttig, Phys. Rev. B 60, 12945 (1999).

${ }^{25}$ D. Schmitz, C. Charton, A. Scholl, C. Carbone, and W. Eberhardt, Phys. Rev. B 59, 4327 (1999).

${ }^{26}$ M. T. Kief and W. F. Egelhoff, Jr., Phys. Rev. B 47, 10785 (1993).

${ }^{27}$ A. Kirilyuk, J. Giergiel, J. Shen, M. Straub, and J. Kirschner, Phys. Rev. B 54, 1050 (1996).

${ }^{28}$ J. Shen, J. Giergiel, A. K. Schmid, and J. Kirschner, Surf. Sci. 328, 32 (1995).

${ }^{29}$ D. A. Steigerwald, I. Jacob, and W. F. Egelhoff, Jr., Surf. Sci. 202, 472 (1988).

${ }^{30}$ W. Daum, C. Stuhlmann, and H. Ibach, Phys. Rev. Lett. 26, 2741 (1988).

${ }^{31}$ H. Glatzel, Th. Fauster, B. M. U. Scherzer, and V. Dose, Surf. Sci. 254, 58 (1991).

${ }^{32}$ S. A. Chambers, T. J. Wagener, and J. H. Weaver, Phys. Rev. B 36, 8992 (1987).

${ }^{33}$ M. Arnott, E. M. McCash, and W. Allison, Surf. Sci. 269/270, 724 (1992). 
${ }^{34}$ Y. C. Lee, H. Min, and P. A. Montano, Surf. Sci. 166, 391 (1986).

${ }^{35}$ D. Pescia, M. Stampanoni, G. L. Bona, A. Vaterlaus, F. Meier, G. Jennings, and R. F. Willis, Phys. Rev. Lett. 60, 2559 (1988).

${ }^{36}$ R. Germer, W. Dürr, J. W. Krewer, D. Pescia, and W. Gudat, Appl. Phys. A: Solids Surf. 47, 393 (1988).

${ }^{37}$ W. A. Macedo, W. Keune, and E. D. Ellerbrook, J. Magn. Magn. Mater. 93, 552 (1991).

${ }^{38}$ D. A. Steigerwald and W. F. Egelhoff, Jr., Surf. Sci. 192, L887 (1987).

${ }^{39}$ A. Schatz, S. Dunkhorst, S. Lingnau, U. von Hörsten, and W. Keune, Surf. Sci. 310, L595 (1994).

${ }^{40}$ J. Thomassen, B. Feldman, and M. Wuttig, Surf. Sci. 264, 406 (1992).

${ }^{41}$ P. Dastoor, M. Arnott, E. M. McCash, and W. Allison, Surf. Sci. 272, 154 (1992).

${ }^{42}$ K. E. Johnson, D. D. Chambliss, R. J. Wilson, and S. Chiang, J. Vac. Sci. Technol. A 11, 1654 (1993).

${ }^{43}$ K. E. Johnson, D. D. Chambliss, R. J. Wilson, and S. Chiang, Surf. Sci. 313, L811 (1994).

${ }^{44}$ D. D. Chambliss and K. E. Johnson, Surf. Sci. 313, 215 (1994).

${ }^{45}$ D. D. Chambliss and K. E. Johnson, J. Vac. Sci. Technol. A 13, 1522 (1995).

${ }^{46}$ J. Giergel, J. Shen, J. Woltersdorf, A. Kirilyuk, and J. Kirschner, Phys. Rev. B 52, 8528 (1995).

${ }^{47}$ A. Brodde and H. Neddermeyer, Surf. Sci. 287/288, 988 (1993).

${ }^{48}$ Th. Detzel, N. Memmel, and Th. Fauster, Surf. Sci. 293, 227 (1993).

${ }^{49}$ N. Memmel and Th. Detzel, Surf. Sci. 307-309, 490 (1994).

${ }^{50}$ H. Jenniches, J. Shen, Ch. V. Mohan, S. S. Manoharan, J. Brathel, P. Ohresser, M. Klaua, and J. Kirschner, Phys. Rev. B 59, 1196 (1999).

${ }^{51}$ K. L. Man, M. S. Altman, and H. Poppa, Surf. Sci. 480, 163 (2001)
${ }^{52}$ The LEED transfer width is related to the inverse of the instrumentally limited diffraction spot width. It is indicative the coherence length of the electron beam and the length scale over which the instrument is sensitive to correlated structures. See, for example, Low Energy Electron Diffraction, edited by M. A. van Hove, W. H. Weinberg, and C. M. Chan (Springer, Berlin, 1986), and references therein.

${ }^{53}$ E. Bauer, Rep. Prog. Phys. 57, 895 (1994).

${ }^{54}$ T. Duden and E. Bauer, J. Electron Microsc. 47, 379 (1998).

${ }^{55}$ W. F. Chung and M. S. Altman, Ultramicroscopy 74, 237 (1998).

${ }^{56}$ M. S. Altman, W. F. Chung, Z. Q. He, H. C. Poon, and S. Y. Tong, Appl. Surf. Sci. 169/170, 82 (2001).

${ }^{57}$ K. Grzelakowski and E. Bauer, Rev. Sci. Instrum. 67, 742 (1996).

${ }^{58}$ T. Duden and E. Bauer, Rev. Sci. Instrum. 66, 2861 (1995).

${ }^{59}$ E. D. Tober, G. Witte, and H. Poppa, J. Vac. Sci. Technol. A 18, 1845 (2000).

${ }^{60}$ L. Hanley, Z. Xu, and J. T. Yates, Jr., Surf. Sci. 248, L265 (1991).

${ }^{61}$ R. Vollmer and J. Kirschner, Phys. Rev. B 61, 4146 (2000).

${ }^{62}$ A. Vaterlaus, C. Stamm, U. Maier, M. G. Pini, P. Politi, and D. Pescia, Phys. Rev. Lett. 84, 2247 (2000).

${ }^{63}$ J. Fassbender, R. Allenspach, and U. Dürig, Surf. Sci. 383, L742 (1997).

${ }^{64}$ J. A. Meyer and R. J. Behm, Surf. Sci. 322, L275 (1995).

${ }^{65}$ F. Nouvertne, U. May, M. Bamming, A. Rampe, U. Korte, G. Guntherodt, R. Pentcheva, and M. Scheffler, Phys. Rev. B 60, 14382 (1999).

${ }^{66}$ X. W. Wang, Surf. Sci. 322, 51 (1995).

${ }^{67}$ Albert Biedemann, Rupert Tschliebnig, Michael Schmid, and Peter Varga, Phys. Rev. Lett. 87, 086103 (2001).

${ }^{68}$ R. K. Kawakami, E. Rotenberg, Ernesto J. Escorcia-Aparicio, Hyuk J. Choi, J. H. Wolfe, N. V. Smith, and Z. Q. Qiu, Phys. Rev. Lett. 82, 4098 (1999). 